\title{
Fault Diagnosis of Gearbox based on the Optimized BP Neural Networks by Improved Particle Swarm Algorithm
}

\author{
Zhimei Duan $^{1, a^{*}}$, Xiaojin Yuan ${ }^{2, b}$ and Yan Xiong ${ }^{3, c}$ \\ ${ }^{1}$ The Engineering College of Honghe University, Mengzi, 661199, China \\ ${ }^{2}$ Comprehensive Test Center, Honghe Quality and Technical Supervision, Mengzi, 661199, China \\ ${ }^{3}$ The Engineering College of Honghe University, Mengzi, 661199, China \\ a704424481@qq.com, b494182567@qq.com, ccjt@163.com
}

Keywords: Gearbox; Adaptive gauss mutation; Particle swarm optimization algorithm; Neural network; Fault diagnosis

\begin{abstract}
In order to improve accuracy of fault diagnosis of gearbox, adaptive mutation particle swarm optimization (AMPSO) algorithm is used to optimize weight of BP. According to fault feature, fault diagnosis is accomplished by optimized BP. The algorithm overcomes disadvantages that slowly convergence and easy to fall into local minima of standard PSO and BP. The simulation results show that the method gains good classification result and has a certain practicality.
\end{abstract}

\section{Introduction}

Rotating Machinery is developing toward the trend of high speed, High power, High reliability and large scale. Once this kind of system failure, it may cause huge loss of personnel and property. So it is very important to guarantee the reliability and security of complex systems. The gear box is the component that reduces speed and increase torque, It is affected by two loading action of torsion and tension-compression, Stress is a complicated process. Gearbox fault rate is about 50\% 70\% in other fault except for engine fault. Since the gear box is a complex transmission, it has complicated nonlinear relations between fault mode and characteristic quantity; moreover, the stochastic factors of gear box in different conditions, experience cannot solve all the problems of diagnosis.

Neural network is an intelligent diagnosis method developed in recent years, widely used in fault diagnosis, and has achieved remarkable results. But standard BP network has the disadvantages that convergence speed is very slow, and convergence precision is not high, even no convergence. The literature [1] uses the improved BP algorithm to diagnosis fault, and improves the convergence speed and searching the global optimal solution, but don't get good effect. The literature [2] use genetic algorithm to improve the neural network weights and threshold values, but it is easy to fall into local optimum, and its coding method is complex.

Particle swarm optimization (PSO) is a kind of swarm intelligence optimization algorithm developed in recent years [3]. It is starting from random solutions, through the iterative search for the global optimal solution. So use it to complete the pre-search can overcome the shortcomings of the BP algorithm, has good robustness. It is a good optimization tools and shows great advantages in practical applications. Still standard PSO algorithm is easy to fall into the same local optimum and has premature convergence phenomenon.

In view of the above problems, in this paper, the fault diagnosis models based on BP neural network structure, introduce an adaptive Gauss mutation particle swarm algorithm to correct network weights, and applied to fault diagnosis of gearbox. Examples results show that the method has good classification effect. And it is suitable for fault diagnosis of gearbox.

\section{Standard BP Network}

Structure of Standard BP Network. BP network is a typical multilayer feed-forward network, is a neural network using error back-propagation training algorithm which proposed by D.E.Rumelhart and J.L.McClelland in 1986 [4]. As long as the appropriate choice of hidden layer node, BP 
network can approximate arbitrary complex nonlinear function. Due to a three layer feed-forward neural network can approach any complex functions, so choose a three layer BP network model [5], shows in Fig. 1.

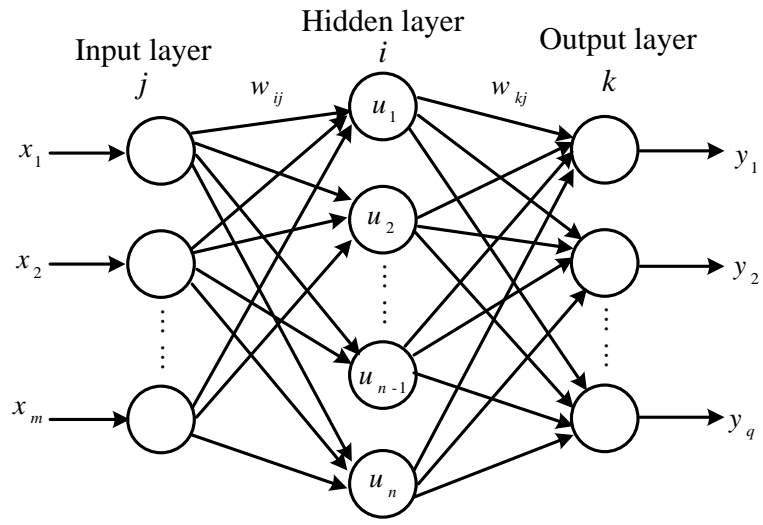

Figure 1. Structure of BP network

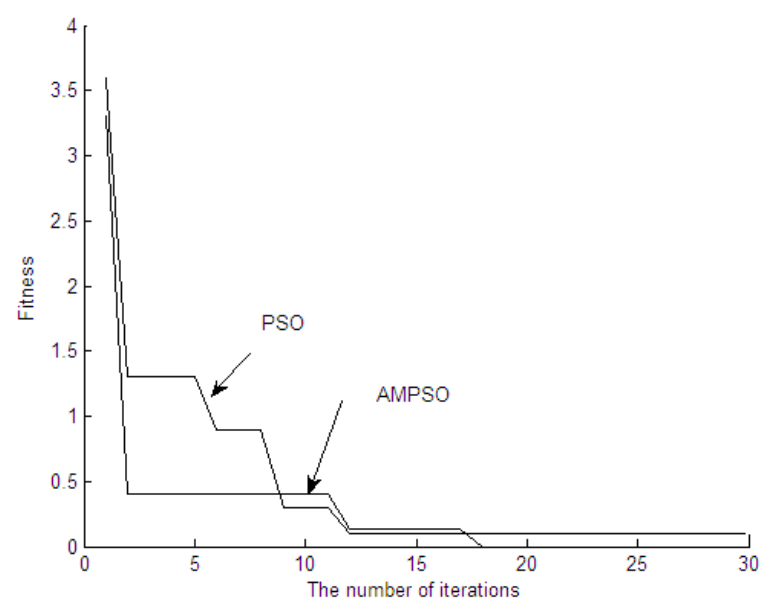

Figure 2. Fitness change curve

In Fig. $1 m, n$ and $q$ are the neurons number of input layer, hidden layer and output layer; $x_{1}$, $x_{2}, \ldots, x_{m}$ is input of input layer, wij is weights between $i$-th neurons of hidden layer and $j$-th neurons of input layer, $w_{k i}$ is weights between $k$-th neurons of output layer and $i$-th neurons of hidden layer, $\mathrm{y} 1, y 2, y q$ is output of output layer.

Learning Algorithm of Standard BP. Suppose $N$ is the training sample, network is trained, using a sample $p$ in the $N$, input of $i$-th neurons of hidden layer is expressed as follow.

$$
n e t_{i}^{p}=\sum_{j=1}^{m} w_{i j} o_{j}^{p}-\theta_{i}=\sum_{j=1}^{m} w_{i j} x_{j}^{p}-\theta_{i}
$$

Which, $i=1,2 \ldots n ; x_{j}^{p}$ and $o_{j}^{p}$ are input and output of $j$-th neurons of input layer when the samples $p$ role, in input layer, $x_{j}^{p}=o_{j}^{p} ; \theta_{i}$ is threshold of $i$-th neurons of hidden layer.

Output of $i$-th neurons of hidden layer is as follow.

$$
o_{i}^{p}=g\left(\text { net }_{i}^{p}\right)
$$

Which, $g(\cdot)$ is activation function.

Input of $k$-th neurons of output layer is as follow.

$$
n e t_{k}^{p}=\sum_{i=1}^{n} w_{k i} o_{i}^{p}-\theta_{k}
$$

Which, $k=1,2 \ldots q ; \theta_{k}$ is threshold of $k$-th neurons of output layer.

Output of $k$-th neurons of output layer is as follow.

$$
o_{k}^{p}=g\left(\text { net }_{k}^{p}\right)
$$

If output is not equal to the desired output $\left(t^{p}\right)$ of the given pattern, the error signal will reverse transmission from the output to input, and in the process of transmission constantly revise weighted coefficient, until obtain the desired output values $\left(t^{p}\right)$ of output layer neurons, after completing the network weights adjustment of sample $p$, And then another sample is entered into learning, until the training of the $N$ sample is completed. Errors function for sample $p$.

$$
J_{p}=\frac{1}{2}\left(t^{p}-o^{p}\right)^{2}
$$

Total error function for all $N$ training samples is as follow.

$$
J=\sum_{p=1}^{N} J_{p}=\frac{1}{2} \sum_{p=1}^{N}\left(t^{p}-o^{p}\right)^{2}
$$




\section{PSO Algorithm and Its Improvement}

Standard PSO Algorithm. Particle swarm optimization algorithm is proposed by Dr. Kennedy and Dr. Eberhart based on bionic swarm optimization algorithm in 1995.it originated from the research groups of bird and fish movement behavior. The basic idea of the PSO algorithm is to find the optimal solution through cooperation and information sharing between the individual groups [6,7].

PSO algorithm first initializes a random particle; find the optimal solution by iteration. Assuming the $d$ dimension of the search space, there are m particles consist of a group. Position and velocity of the $i$-th particle is divided into : $X_{i}=\left(x_{i 1}, x_{i 2}, \ldots, x_{i d}\right)$ and $V_{i}=\left(v_{i 1}, v_{i 2}, \ldots, v_{i d}\right)$.In each iteration, the particles by tracking two optimal solutions to update their own: the first one is the particle itself to find the optimal solution, that individual extreme value $\left(P_{\text {best }}\right)$.Another is the optimal solution to be found among the entire population, that global optimal solution $\left(p_{\text {gbest }}\right)$. find two optimal solution, the particles according to the following formula to update their own speed and position is as follow.

$$
\begin{aligned}
& v_{i d}(k+1)=w v_{i d}(k)+c_{1} r_{1}\left(p_{i d}(k)-x_{i d}(k)\right)+c_{2} r_{2}\left(p_{g d}(k)-x_{i d}(k)\right) \\
& x_{i d}(k+1)=x_{i d}(k)+v_{i d}(k+1)
\end{aligned}
$$

Which, $w$ is inertia weight, it makes the particle keep moving inertia; $c_{1}$ and $c_{2}$ is acceleration factor; $k$ is the number of iterations; $r_{1}$ and $r_{2}$ is random number, distributed in the interval [0,1].

Practice shows that, if the $w$ decreases linearly with the conduct of the iterative algorithm, will be able to significantly improve the convergence performance of the algorithm. Often use the following equation.

$$
w=w_{\max }-\frac{k *\left(w_{\max }-w_{\min }\right)}{k_{\max }}
$$

Which $w_{\max }$ and $w_{\min }$ are maximum value and minimum value of the $w, w_{\max }=0.9, w_{\min }=0.4 ; k$ is current iteration step; $k_{\max }$ is the maximum value and the minimum value.

Adaptive Mutation Particle Swarm Optimization (AMPSO). In the iterative process, all particles of Standard PSO algorithm are closer to the optimal position. If the optimal location is a local optimum, particle swarm cannot re-search in the solution space and fall into the local optimum. By this time, the mutation operation is carried out to change the global extreme $p_{\text {gest }}$, the forward direction of the particle can be changed and get into other regions continue to search. Again and again, the algorithm can find the global optimal. This is the basic idea of adaptive mutation mechanism [8].In order to enhance the global search ability of the particle swarm, mutational operating on one-dimensional of random selection with a certain probability, value of mutation probability $p$ is as follow.

$$
p=\left\{\begin{array}{c}
k, \sigma^{2}<\sigma_{d}^{2} \quad \text { and } f\left(p_{\text {gbest }}\right)>f_{d} \\
0, \quad \text { other }
\end{array}\right.
$$

Which $k$ is arbitrary values of the $[0.1,0.3] ; \sigma^{2}$ is he groups adapt degrees variance; $\sigma_{d}^{2}$ is generally take the maximum value that much less than the $\sigma^{2}, f_{d}$ is expected optimal solution.

Let $p_{\text {gbest }}^{k}$ be the k-dimensional value of $p_{\text {gbest }}$, use the method of increasing random perturbations to mutation operation $p_{\text {gbest }}^{k}, \eta$ is random variables obey gauss $(0,1)$ distribution. Often use the following equation.

$$
p_{\text {gbest }}^{k}=p_{\text {gbest }}^{k}(1+0.5 \eta)
$$

\section{Realization of Adaptive Mution Particle Swarm Neural Network Algorithm}

In paper, based on BP model has been established, combine adaptive mutation particle swarm optimization with BP algorithm, AMPSO is used to search initial weights and threshold of BP model, then BP network is used to optimize the initial weights and thresholds for secondary, and obtain the final weights and threshold.AMPSO [9,10] algorithm steps are as follows.

Step 1; determine the structure of network based on training sample set of the input and output. 
Step 2; determine the particle population size, particle initialization position, speed, inertia weight, learning factor and the number of iterations.

Step 3, determine the fitness function.

$$
\text { fitness }=\frac{1}{N} \sum_{i=1}^{N}\left(y_{i}-d_{i}\right)^{2}
$$

Which $N$ is the number of samples for training; $y_{i}$ is the actual network output of the $i$-th sample; $d_{i}$ is the desired output of the $i$-th sample.

Step 4, calculate the fitness of each particle by type (12), network activation function is sigmoid.

Step 5; compare the fitness value with the experienced best position $p_{i}$, if it is good, as the best current position $p_{i}$. For the global extreme value, compare the fitness value with the experienced best position $p_{g}$, if it is good, regard the current fitness value of the particle as the groups' globally optimal fitness value, and set $p_{g}$ to the current position of the particle.

Step 6, update velocity and position by type (7) (9).

Step 7, Calculate mutation probability $p$ by type (10); perform mutation operating by type(11).

Step 8, if the number of iterations reaches a given maximum times or reaches the minimum error requirement, then stop the iteration. At this time, $p_{g}$ corresponding value is weights and thresholds of BP network, put weights and threshold into the BP network for secondary learning and training, eventually form the fault diagnosis model of transformer. Otherwise go to step 3 continue iteration.

\section{Gearbox Fault Diagnosis Based on AMPSO}

Extracting Feature Vector of Gearbox. The gear box of engine as the research object, in the gear box failure parts, the proportion of gear failure is the largest, about $70 \%$, and bearing followed, so focus of gearbox fault diagnosis is the gear and bearing fault. The common failure forms of gear: tooth fracture, tooth surface wear, tooth surface fatigue and tooth surface plastic deformation. The common failure forms of bearing: flaw, denudation and indentation. In this paper, the fault diagnoses of gearbox vibration test, and obtain the vibration signal of each test point by piezoelectric acceleration sensor which installed each sensitive point in the box, use data acquisition and data processing system to monitor engine gearbox vibration state, put the gear and bearing magnitude as the criterion to transmission system fault or not, select 8 test points as detection object. Define 3 kinds of gear fault modes: fault-free $(1,0,0)$; root $\operatorname{crack}(0,1,0)$; tooth broken $(0,0,1)$.

Fault Diagnosis of Gearbox. According to the 135 groups data collected, selected 95 groups as training samples, the other 40 groups as test sample. In experiments, BP network structure is 8-17-3, AMPSO algorithm parameter: Population size $20, c_{1}=c_{2}=15$, Inertia weight $w$, with the number of iterations linearly decreases from 0.9 to 0.3 , the maximum number of iterations is 30 , mutation probability $p=0.2$. Fig. 2 is the fitness value change curve that the PSO and AMPSO two algorithms stop iterating when variance equal to 0.001 .

As can be seen, PSO algorithm in the iteration of 12 times has been caught in a local optimum. For AMPSO algorithm, due to the increase in the mutation operation, making the particle into the local optimum into other regions continues to search. Reduce the speed of the algorithm convergence to enhance the local convergence ability and improve the training of the neural network performance.

According to the selected training samples, the algorithm of AMPSO-BP and PSO-BP are used to the neural network of same structure for learning and training. Results are shown in Tab.1.it can be seen from Tab.1, fault identifying rate of AMPSO-BP algorithm is the highest; it is effective to diagnose the fault of gearbox. Tab. 2 lists the 6 typical examples of gearbox fault samples.

\section{Conclusions}

The AMPSO algorithm is applied to the BP neural network, algorithm is simple and easy to implement. Only need the sample data must be able to deal with optimization problems, which has wide applicability. It overcomes the defect of the standard PSO algorithm is easy to fall into local 
minima, effectively avoids the error of fault diagnosis, improves the accuracy of fault diagnosis, There is certain reference value in practice.

Table 1 Comparision of diagnosis results

\begin{tabular}{|c|c|c|c|c|c|c|c|}
\hline \multirow{2}{*}{$\begin{array}{c}\text { fault } \\
\text { type }\end{array}$} & \multirow{2}{*}{$\begin{array}{c}\text { testing } \\
\text { sample }\end{array}$} & \multicolumn{6}{|c|}{ fault diagnosis } \\
\cline { 3 - 6 } & & \multicolumn{2}{|c|}{ positive number } & \multicolumn{2}{c|}{ positive rate/\% } & \multicolumn{2}{c|}{ Average positive rate/\% } \\
\cline { 3 - 6 } & AMPSO-BP & PSO-BP & AMPSO-BP & PSO-BP & AMPSO-BP & PSO-BP \\
\hline 100 & 13 & 12 & 11 & 92.3 & 84.6 & \\
\hline 010 & 12 & 11 & 10 & 91.6 & 83.3 & \multirow{2}{*}{92.4} & 84.9 \\
\hline 001 & 15 & 14 & 13 & 93.3 & 86.7 & & \\
\hline
\end{tabular}

Table 2 Diagnosis sample of AMPSO

\begin{tabular}{|c|c|c|c|c|c|c|c|c|c|c|}
\hline No. & $\begin{array}{c}\text { testing } \\
1\end{array}$ & $\begin{array}{c}\text { testing } \\
2\end{array}$ & $\begin{array}{c}\text { testing } \\
3\end{array}$ & $\begin{array}{c}\text { testing } \\
4\end{array}$ & $\begin{array}{c}\text { testing } \\
5\end{array}$ & $\begin{array}{c}\text { testing } \\
6\end{array}$ & $\begin{array}{c}\text { testing } \\
7\end{array}$ & $\begin{array}{c}\text { testing } \\
8\end{array}$ & $\begin{array}{c}\text { actual } \\
\text { fault }\end{array}$ & diagnosis results \\
\hline 1 & 0.2711 & 0.1230 & 0.0720 & 0.2922 & 0.0947 & 0.1147 & 0.5157 & 0.2306 & $(1,0,0)$ & $(0.9985,0.0014,0.0025)$ \\
\hline 2 & 0.0563 & 0.2542 & 0.1319 & 0.5157 & 0.1298 & 0.0753 & 0.0438 & 0.1590 & $(1,0,0)$ & $(0.9987,0.0021,0.0005)$ \\
\hline 3 & 0.0058 & 0.1803 & 0.0992 & 0.0802 & 0.1002 & 0.1823 & 0.1112 & 0.0412 & $(0,1,0)$ & $(0.0042,0.9986,0.0013)$ \\
\hline 4 & 0.1159 & 0.0090 & 0.1453 & 0.1911 & 0.1506 & 0.1640 & 0.1852 & 0.2718 & $(0,1,0)$ & $(0.0027,0.9975,0.0038)$ \\
\hline 5 & 0.0522 & 0.0882 & 0.1818 & 0.0871 & 0.0500 & 0.1503 & 0.1680 & 0.0450 & $(0,0,1)$ & $(0.0014,0.0023,0.9991)$ \\
\hline 6 & 0.2235 & 0.1171 & 0.0683 & 0.2597 & 0.1167 & 0.1002 & 0.2281 & 0.2668 & $(0,0,1)$ & $(0.0042,0.0003,0.9979)$ \\
\hline
\end{tabular}

\section{References}

[1] YANG Shu-lian.Application of BP neural network in fault diagnosis of gearbox.MACHINE TOOL \& HYDRAULICS,2006,(7):244-246.

[2] DENG Hong-gui, CAO Jian. A novel genetic algorithm and its application to transformer fault diagnosis [J].J.CENT.SOUTH UNIV.(SCIENCE AND TECHNOLOGY), 2005, 36(3):481-484

[3] LU Ning, ZHOU Jian-zhong, HE Yao-yao. Particle swarm optimization-based neural network model for short-term load forecasting [J]. Power System Protection and Control, 2010, 38(12): 65-68.

[4] SHI Biao, LI Yu-xia. Short-term load forecast based on modified particle swarm optimizer and back propagation neural network model [J]. Journal of Computer Applications, 2009, 29(4):1036-1039.

[5] Zhexue Ge,Zhiqiang Sun:Theory of Neural Network and Matlab R2007(Electronics Industry Publications, China 2007),p.108-116.

[6] CHENG Hui-jie, ZHANG Guo-yin, HE Ying. Study of Tumor Classification Based on Particle Swarm Neural Network Ensemble [J].Computer Engineering, 2010, 36(10):209-211.

[7] KOU Pan-gao, ZHOU Jian-zhong, HE Yao-yao, et al, Optimal PID Governor Tuning of Hydraulic Turbine Generators With Bacterial Foraging Particle Swarm Optimization Algorithm, Proceedings of the CSEE, 29(26) (2009) 101-106.

[8] ZHAO Ji-yin, LI Jian-po, ZHENG Rui-rui. Application of Adaptive Learning Rate Method inPower Transformer Fault Diagnosis [J]. Journal of Jilin University (Information Science Edition), 2008, 26(4):415-419.

[9] FU Shao-chang, HUANG Hui-xian, XIAO Ye-wei, WU Yi, WANG Chen-hao. Application of Adaptive Mutation-particle Swarm Optimization Algorithm in Traffic Control [J]. Journal of System Simulation, 2007, 19(7):1562-1564. 
[10] WANG Xiao-xia, WANG Tao. Power Transformer Fault Diagnosis Based on Neural Network Evolved by Particle Swarm Optimization [J].High Voltage Engineering, 2008,34(11):2362-2367. 